\title{
Experimental Study on the Measuring Temperature Field by Real-Time Digital Holography
}

\author{
Zhan-Rong ZHOU ${ }^{*}$, Xiao-Fang SHEN, Ai-Jun LI \\ Department of Physics, Xi'an High Technology Studio, Xi'an 710025, China \\ aemail: zhouzhou76@163.com
}

\begin{abstract}
Keywords: measuring temperature field ;digital holographic; interferometry; Fourier transform Abstract. An experimental method and reconstruction arithmetic of temperature field are proposed. The fast Fourier transform followed by a digital low-pass filter and the inverse Fourier transform were applied to interference fringes for removing speckle noise, so that airflow field phase information is in high-contrast and low noise. Phase was unwrapped to calculate phase difference. The temperature distribution was obtained by the relation between phase and refractive index. The results are consist with the values measured by thermocouple. It is shown that the digital holographic interferometry is an effective method for measuring temperature field,so that the measuring precision can be greatly improved.
\end{abstract}

\section{Introduction}

Digital holography is a novel imaging technique of optical holography, computer image processing and electronic record combination. It has the advantages of digital processing, quantitative analysis of phase field and real-time, and has become a research hotspot in the field of optical information processing. With the appearance of high-performance CCD and the rapid development of computer technology, digital holographic interferometry shows more powerful vitality. Real time digital holographic interferometry has the advantages of full field, no interference, non-contact, high measurement precision and 3D visual measurement. Some domestic and foreign scholars have done a lot of work in this field and have made great progress. The application of digital holography has been extended to the particle field measurement, shape measurement, vibration measurement, physical properties of materials testing, biological detection etc. But in actual measurement, the measured physical quantities are recorded in the form of interference fringes. How to extract the measured data from the interference fringe image is a difficulty to restrict the development and application of digital holography[1-3].

Digital holography is applied to the temperature measurement of gas flow field in this paper. The corresponding temperature distribution are extracted from the fringe pattern of temperature field. In the actual temperature field measurement, many objects can be equivalent to two-dimensional axisymmetric distribution, such as internal combustion airport, combustion field, aircraft flow field, etc $[4,5]$. Therefore, it is of practical significance to study the measurement of the two-dimensional axisymmetric temperature field.

\section{Measuring principle}

Digital holography inherits the basic idea of ordinary optical holography[6]. The biggest feature is to replace CCD as holographic recording medium. The recorded holograms are stored in the computer after digital processing, and then to the digital Fourier transformation replaces optical diffraction to achieve record field reconstruction. Through the Fourier transform of the recorded hologram intensity distribution, the spatial spectrum distribution is obtained. If a coherent plane wave through a medium temperature fluctuation space, the optical transmission medium will carry information by irradiation area temperature distribution. The complex amplitude distribution of the light wave passing through the temperature field can be reproduced when the hologram recorded the temperature field varies at different time[7,8].

Assuming that the complex amplitude distribution of the light wave before temperature field changes: 


$$
O(x, y)=O_{0}(x, y) \exp \left[j \phi_{0}(x, y)\right]
$$

In the formula(1), the $O_{0}(x, y)$ is the amplitude distribution, and the $\phi_{0}(x, y)$ is phase distribution. When the temperature field changes, the refractive index distribution of the medium changes, so that the phase distribution of the light wave is changed, but the amplitude generally does not change. Therefore, the complex amplitude distribution of the light wave can be expressed as:

$$
O^{\prime}(x, y)=O_{0}(x, y) \exp \left[j \phi_{0}^{\prime}(x, y)\right]
$$

The two holograms recorded before and after the temperature field are added together, and then the digital Fourier transform and the filtering process are used to reproduce the complex amplitude $O(x, y)$ and $O^{\prime}(x, y)$. The expression of superposition intensity distribution is expressed as :

$$
I(x, y)=\left|O(x, y)+O^{\prime}(x, y)\right|^{2}=4 O^{2}{ }_{0}(x, y) \cos ^{2}\left[\frac{\phi_{0}^{\prime}(x, y)-\phi_{0}(x, y)}{2}\right]
$$

The formula shows that if the two temperature fields are different, the interference fringes will appear in the reconstructed intensity distribution image.

The interference fringe is transformed by Fourier. The +1 level spectrum is extracted with the design suitable digital low pass filter, and then transformed by inverse Fourier transform, and then the unwrapping phase is processed. The true reflection of wave shape deformation can be got.

If there is the shape of the wavefront deformation by the flow field, the phase change value $\Delta \phi(r)$ of the measured object wave at certain position can be obtained:

$$
\Delta \phi(r)=\frac{2 \pi}{\lambda} \int_{0}^{L}\left[n(r)-n_{0}\right] d r
$$

In the formula (4), the $\mathrm{L}$ is the length of the test section, the $n_{0}$ is the original refractive index distribution to be measured. The $n(r)$ is the refractive index distribution of the measured temperature field after the phase change.

By means of mathematical inversion of the formula (4), the refractive index distribution in the measured temperature field can be determined $n(r)$.

To the uniform and transparent media field, It can obtain that the refractive index is a function of medium density according to the classical electrodynamics knowledge. That is G-D formula:

$$
\frac{n-1}{\rho}=K
$$

In the formula (5), the $\rho$ is the medium density, the $\mathrm{K}$ is a G-D constant[9].

Pressure is generally considered to be constant in the temperature distribution of gas flow field, and its density and temperature are similar to the state equation of ideal gas:

$$
\rho=\frac{M P}{R T}
$$

In the formula (6), the $\mathrm{P}$ is the gas pressure, the $\mathrm{T}$ is the temperature, the $\mathrm{M}$ is the gas molecular weight, the $\mathrm{R}$ is the gas constant.

In the temperature distribution test of the gas flow field, the refractive index change of gas disturbance is obtained by the interference fringes. The relationship between the refractive index and temperature is derived from the formula (6) and formula (7).

$$
n(r)-1=\frac{M P K}{R T}
$$

The change between temperature and refractive index can be obtained:

$$
T(r)=\frac{M P T}{R\left[\Delta n(r)+n_{0}-1\right]}
$$


The formula (8) reveals the relationship between the refractive index and temperature field, which is the theoretical basis for measuring the temperature of the digital holographic interferometry.

\section{Experimental apparatus and results}

The experimental light path is shown in Figure 1. The laser beam emitted by He-Ne laser, which is divided into two beams by the BS1.A beam of light passes through the temperature field to be measured, and then the light is projected onto the CCD light sensitive surface by the total reflection mirror M1 and the beam splitter mirror BS2.A The other beam is projected on the CCD light sensitive surface by the total reflection mirror M2 and the beam splitter mirror BS2 as the reference light. The reference light and the object light are adjusted to a certain angle by the beam splitter mirror BS2 to form an interference pattern on the CCD photosensitive surface. The CCD resolution is $795 \times 596$ array. The pixel size is $0.010 \mathrm{~mm} \times 0.0108 \mathrm{~mm}$.

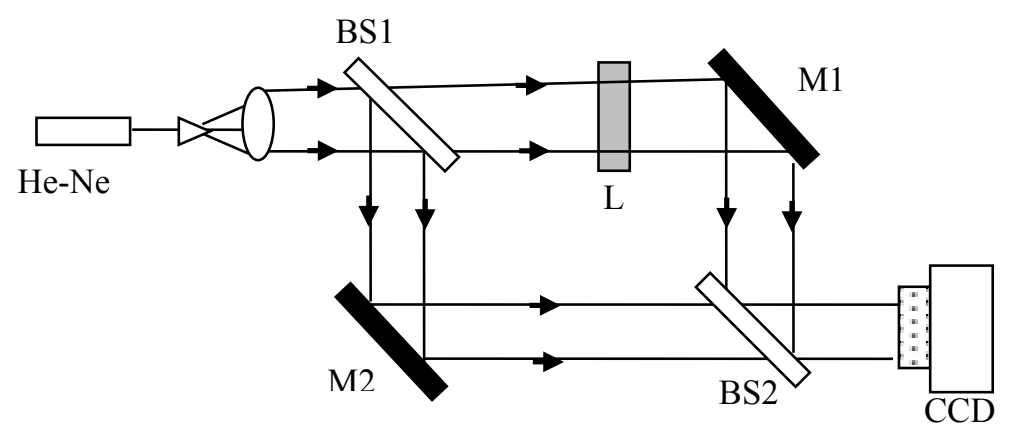

Fig. 1 Experimental setup for digital holographic interferometry

The measured temperature field is an air temperature field which is formed by the hot wire with current. In the experiment, the temperature field is kept stable, so the temperature field has axial symmetry. A sequence of interference patterns are collected, as shown in Figure 2. This set of interference patterns reflects the change of the temperature around the object. The local temperature is the same refractive index, and the optical path is the same. So each fringe is an isotherm. The distribution of interference fringe pattern is only determined by the phase distribution of the temperature change object wave. The phase difference of each point can be directly obtained by using the formula (7) and the formula (8). So that the temperature of the each pixel space position in the field can be calculated accurately.

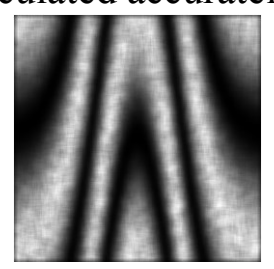

( a ) $\mathrm{t}=10 \mathrm{~s}$

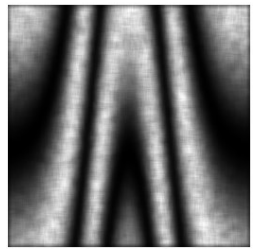

( b ) $\mathrm{t}=20 \mathrm{~s}$

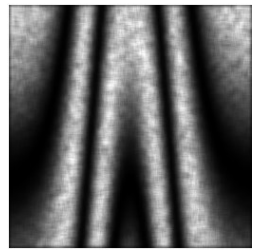

( c ) $\mathrm{t}=30 \mathrm{~s}$

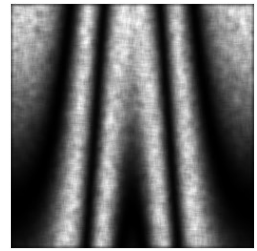

( d ) $\mathrm{t}=40 \mathrm{~s}$

Fig. 2 Experimental results of digital holographic interferometry

\section{Data extraction and verification of interference fringe pattern}

The interference fringe images were transformed by Fourier transform. The +1 spectrum were extracted by the suitable digital low-pass filter. The wrapped phase map as shown in Figure 3 can be got by the inverse Fourier transform . The wrapped phase is performed by the means of the authors in the literature [10], as shown in Figure 4. 


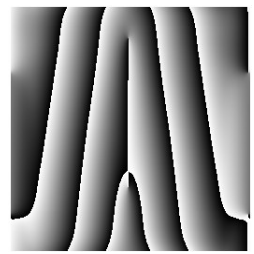

( b ) $\mathrm{t}=20 \mathrm{~s}$

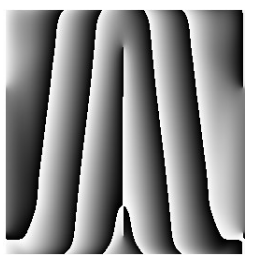

( c ) $\mathrm{t}=30 \mathrm{~s}$

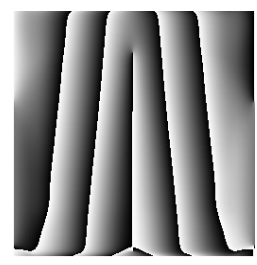

( d ) $\mathrm{t}=40$ s

Fig. 3 Wrapped phase distribution

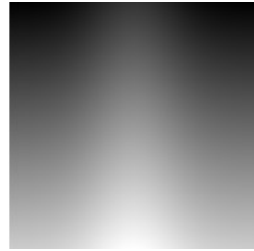

( a ) $\mathrm{t}=10 \mathrm{~s}$

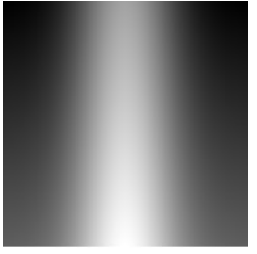

( b ) $\mathrm{t}=20 \mathrm{~s}$

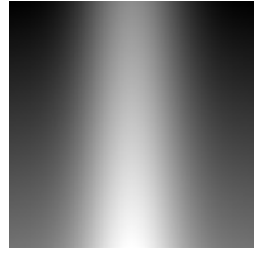

( c ) $\mathrm{t}=30 \mathrm{~s}$

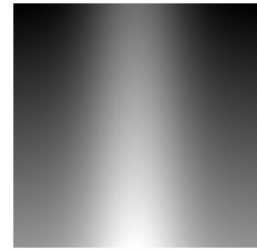

( d ) $\mathrm{t}=40 \mathrm{~s}$

Fig. 4 Unwrapping phase distribution

The laser wavelength is $\lambda=632 \mathrm{~nm}$, and the ambient temperature is $18.5{ }^{\circ} \mathrm{C}$. The temperature distribution is obtained by the formula (8) as shown in Figure 5. Because of the frequency domain filtering in phase extraction, the speckle noise and other high frequency noises are naturally processed, so the method has the ability of anti noise.

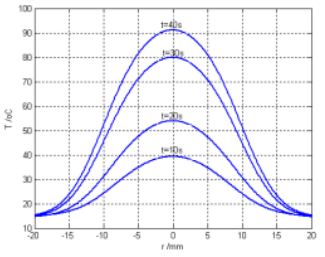

Fig. 5 Temperature distribution

In order to verify the correctness of real-time digital holography measurement results, we keep the whole experimental device not to move, and return to the initial state of the environment. The thermocouples temperature is fixed at the distance of $10 \mathrm{~mm}$ from the resistance wire. Respectively, at $10 \mathrm{~s}, 20 \mathrm{~s}, 30 \mathrm{~s}, 40 \mathrm{~s}$, the measured results were $23.3{ }^{\circ} \mathrm{C}, 29.6{ }^{\circ} \mathrm{C}, 40.1{ }^{\circ} \mathrm{C}, 52.3{ }^{\circ} \mathrm{C}$. Consistent with the proposed method, the temperature field of digital holography is an effective method.

\section{Conclusion}

In this paper, the digital holographic interferometry is introduced into the measurement of airflow temperature field. The relationship between the phase of the optical field, the refractive index of the gas flow field and the temperature of the air flow field is analyzed. The phase distribution of the light field is obtained from the interference fringe by Fourier transform, and the temperature distribution of the airflow field is obtained quantitatively. The holographic measurement results are in agreement with the actual temperature. The method provides an effective means for measuring temperature field. The method not only makes the invisible temperature field appear on the computer in the form of interference fringe image, but also has the advantages of real-time observability, high measurement accuracy, no interference and good development and application prospect.

\section{References}

[1] L. Miccio, D. Alfieri, S. Grilli et al.. Direct full compensation of the aberrations in quantitative phase microscopy of thin objects by a single digital hologram[J]. Appl. Phys. Lett. ,90(2007):041104.

[2] P. Ferraro, G. Coppola, D. Alfieri et al.. Controlling images parameters in the reconstruction process of digital holograms[J]. IEEE,J.Selected Topics in Quant. Electron. 10(2004):829 839. 
[3] L. Xu, X. Y. Peng, J.M. Miao et al..Studies of digital microscopic holography with application to microstructure testing[J].Appl. Opt. 40(2001):5046 5051.

[4] S. Nakadate, M. Isshiki. Real-time vibration measurement by a spatial phase-shifting technique with tilted holographic interferogram [J]. Appl. Opl.36(1997): 281 284.

[5] Di Jianglei, Zhao Jianlin, Fan Qi et al..Phase correction of wavefront reconstruction in digital holographic microscopy[J]. Acta Optica Sinica,28(2008):57 61.

[6] Lin Qizhao. Investigation on measuring the field of temperature and concentration in a diesel spray by laser double exposure holographic interferometry method[J]. Applied Laser, 18(1998):171 174.

[7] Zhang Peng, Zhou Yingbiao, Zheng Chuguang. Reconstructing ax-symmetric temperature field by real-time holographic interference measuring method[J]. Power Engneerng, 23(2003):2321 2324.

[8] Zhao Jianlin, Tan Haiyun. Measuring three-dimensional temperature field by digital holographic interferometry[J]. Acta Optica Sinica, 22(2002):1447 1451.

[9] C. M. Vist. Holographic Interferometry [M]. Beijing: Mechanical Industry Press, 1984. pp.

$118 \sim 124$.

[10] Zhou Zhanrong Wang Hongxia, Ma Jin. An improved phase unwrapping algorithm[J]. Information Electronic and Computer Science,11(2010):423 426. 